\title{
Improved heart failure by Rhein lysinate is associated with p38MAPK pathway
}

\author{
WEI WANG, XIN MENG, JING WANG and YONG LI \\ Internal Medicine-Cardiovascular Department, Tengzhou Central People's Hospital Affiliated \\ to Jining Medical University, Zaozhuang, Shandong 277500, P.R. China
}

Received February 26, 2017; Accepted March 26, 2018

DOI: $10.3892 /$ etm.2018.6423

\begin{abstract}
The present study aimed to explore the role of Rhein lysinate (RHL) in neonatal rat ventricular myocytes (NRVMs) and congestive heart failure induced by co-arctation of the abdominal aorta. Male Sprague-Dawley rats were divided into 3 groups randomly: co-arctation of abdominal aorta group (A group, $n=10)$, sham operation group ( $\mathrm{SH}$ group, $\mathrm{n}=10$ ) and RHL treatment rats (A+RHL group, $n=10)$. To establish an in vitro oxidative stressed cardiomyocyte model, NRVMs were treated with $10 \mu \mathrm{M} \mathrm{H}_{2} \mathrm{O}_{2}$ for $24 \mathrm{~h}$. MTT assay indicated that $\mathrm{H}_{2} \mathrm{O}_{2}$ treatment reduced primary cardiomyocyte viability in a time- and dose- dependent manner, whereas RHL abolished the detrimental effects of $\mathrm{H}_{2} \mathrm{O}_{2}$, indicating a protective role of RHL. Further study demonstrated that $\mathrm{H}_{2} \mathrm{O}_{2}$-induced reactive oxygen species (ROS) production was reversed by RHL. Then, TUNEL staining was carried out and the results revealed that $\mathrm{H}_{2} \mathrm{O}_{2}$ markedly enhanced primary cardiomyocyte apoptosis. Conversely, RHL incubation decreased $\mathrm{H}_{2} \mathrm{O}_{2}$-induced cell apoptosis, indicating the protective role of RHL in primary cardiomyocytes. Furthermore, abnormal p38 activation was identified in the failed heart. Notably, treatment with RHL reduced p38 activation. In addition, RHL significantly enhanced the expression of anti-apoptotic protein, B cell lymphoma $(\mathrm{Bcl})-2$, however markedly reduced the protein level of $\mathrm{Bcl}-2$ associated $\mathrm{X}$, apoptosis regulator in primary cardiomyocytes, indicating its anti-apoptotic role in the cardiac setting. Overall, RHL protects heart failure primarily by reducing ROS production and cardiomyocyte apoptosis via suppressing p38 mitogen activated protein kinase activation.
\end{abstract}

Correspondence to: Dr Wei Wang, Internal MedicineCardiovascular Department, Tengzhou Central People's Hospital Affiliated to Jining Medical University, 89 Guhui Road, Zaozhuang, Shandong 277500, P.R. China

E-mail: wangwei17230@163.com

Key words: Rhein lysinate, heart failure, apoptosis, p38MAPK

\section{Introduction}

Cardiovascular disease is one of the most common non-cancer-related death and disability in the world $(1,2)$. The mortality of acute myocardial infarction has been decreased in the past years (3). However, heart failure-(HF-) induced mortality is still increasing due to the deteriorating cardiac contractile function and left ventricular remodeling $(4,5)$. Unfortunately, poor knowledge is known about the mechanism of metabolic dysfunction in HF.

p38MAPK signaling is suggested to play a key role in the stressed cardiomyocytes (6-8). It is reported that oxidative stress or ischemia reperfusion could activate the p38MAPK, thereby inducing cardiomoycyte apoptosis (9). Studies have shown that inhibition of p38MAPK could improve inflammatory reactions and protect cardiomyocytes from apoptosis $(6,7)$.

The application of herbal medicines is common in Asian countries due to the lower adverse effects and effectiveness in various human diseases (10). Rhein is isolated from the rhizome of rhubarb and is characterized by broad pharmacological effects, including antidiabetic activity, anti-inflammation and inhibition of interleukin-1-induced chondrocyte activation $(11,12)$. However, its application is largely limited due to the poor water insolubility. And Rhein lysinate (RHL) is modified with lysine, which is water soluble in drinking water $(11,12)$. However, whether RHL could improve the cardiac function after HF has never been explored.

In the current study, we first explored the protective role of RHL in HF. Our data showed that RHL could inactivate p38MAPK signaling in cardiomyocytes, thereby protecting cardiac function from HF.

\section{Materials and methods}

Experimental animals. A total of forty 11-14 week-old male healthy Sprague-Dawley rats were obtained from Experimental Animal Center of Tengzhou Central People's Hospital affiliated to Jining Medical University. They were divided into 3 groups randomly: coarctation of abdominal aorta group (A group, $n=20$ ) sham operation group ( $\mathrm{SH}$ group, $\mathrm{n}=10)$ and control group (C group, $\mathrm{n}=10)$. Laparotomy was performed after anesthesia by intraperitoneal injection of $3 \%$ pentobarbital sodium. Abdominal artery was stripped at approximately $5 \mathrm{~mm}$ from the above left renal artery opening, 
a 6/0 silk suture was tied around and made up to $65-70 \%$ constriction of abdominal aorta. RHL treatment rats (A+RHL group) were pretreated with RHL $(1.5 \mathrm{~g} / \mathrm{kg})$ for 3 days by gavage for 14 additional days. Sham operated animals underwent the same procedure except the ligation. Housing and procedures involving experimental animals were in accordance with the Guide for the Care and Use of Tengzhou Central People's Hospital affiliated to Jining Medical University. All animal experiments were approved by the Animal Care and Studies committee of Tengzhou Central People's Hospital affiliated to Jining Medical University.

Preparation of RHL. RHL was purchased from the Shi-Feng Biological Co., Shanghai, China. The RHL was dissolved in PBS to $10 \mathrm{mg} / \mathrm{ml}$ and then diluted with DMEM culture medium containing $10 \% \mathrm{FBS}$ at different concentrations.

Echocardiography. Rats were lightly anesthetized with 1-1.5\% isolurane in oxygen until the heart rate stabilized to 400 to 500 beats per minute. Echocardiography was carried out with Vevo 770 and Vevo 2100 (VisualSonics) instruments. Fraction shortening (FS), ejection fraction (EF), let ventricular internal diameter (LVID) during systole, LVID during diastole, end-systolic volume, and end-diastolic volume were calculated with Vevo Analysis software (version 2.2.3). After that, rats were euthanatized by cervical dislocation, and their hearts were collected for further analyses.

Histology, immunoluorescence, and immunohistochemistry. Heart tissues were cut into cryosections and subsequently analyzed by H\&E staining according to the manufacturer's protocol (Sigma-Aldrich). For the histological analysis, $8 \mu \mathrm{m}$ sections were incubated with primary antibodies overnight at $4^{\circ} \mathrm{C}$. Then, the sections were washed with $0.25 \%$ Triton X-100 in PBS and incubated with either fluorescently labeled (Molecular Probes; Invitrogen) or biotinylated secondary (Vector) antibodies for $2 \mathrm{~h}$. Then, the sections were observed using microscopy.

Terminal deoxynucleotidyl transferase-mediated dUTP nick end labeling (TUNEL) staining. Nuclear fragmentation was detected by TUNEL staining with an apoptosis detection kit (Roche) or by incubating fixed cells using an apoptosis detection kit (R\&D Systems) according to the manufacturer's protocol. Cells (500-700) in 10 randomly chosen fields from each dish were counted to determine the percentage of apoptotic nuclei. Each data point indicates results from 1,600 to 2,000 cells from 4 independent experiments.

Isolation and culture of rat cardiac myocytes. Neonatal rat ventricular myocytes (NRVMs) were isolated from 1-3-day-old Sprague Dawley rats via combined $0.2 \%$ trypsin and $0.1 \%$ collagenase type II digestion. The cardiac myocytes were plated at a density of $6.6 \times 10^{4}$ cells $/ \mathrm{cm}^{2}$ in DMEM supplemented with $10 \%$ FBS supplemented with $0.1 \mathrm{mM}$ 5-bromo-2-deoxyuridine. Fibroblasts were not detected in these cultures as determined by immunocytochemical staining with an anti-fibronectin antibody.

Protein extraction and western blot analysis. Proteins samples were extracted from cardiomyocytes or myocardial tissue in RIPA buffer (1\% TritonX-100, $15 \mathrm{mmol} / \mathrm{l} \mathrm{NaCl}$, $5 \mathrm{mmol} / 1$ EDTA, and $10 \mathrm{mmol} / \mathrm{l}$ Tris- $\mathrm{HCl}$ (pH 7.0) (Solarbio, China) supplemented with a protease and phosphatase inhibitor cocktail (Sigma) and then separated by 10\% SDS-PAGE, followed by electrophoretic transfer to a PVDF membrane. After soaking with $8 \%$ milk in PBST ( $\mathrm{pH} 7.5$ ) for $2 \mathrm{~h}$ at room temperature, the membranes were incubated with the following primary antibodies: anti-p-p38, anti-p38, Bcl-2, Bax and anti-GAPDH (Cell signaling). Immunodetection was performed by enhanced chemiluminscence detection system (Millipore) according to the manufacturer's instructions. The house-keeping gene GAPDH was used as the internal control.

MTT assay. NRVMs (5,000 cells/well) were plated in 24-well plates and pretreated with RHL for $1 \mathrm{~h}$ and then treated with the indicated concentrations of $\mathrm{H}_{2} \mathrm{O}_{2}$ for $24 \mathrm{~h}$. All assays were carried out in triplicate. The cells were incubated with $0.5 \mathrm{mg} / \mathrm{ml}$ 3-[4,5-dimethylthiazol-2-yl]-2,5-di-phenyl-tetrazolium bromide for $4 \mathrm{~h}$. And the relative fluorescence was determined at $490 \mathrm{~nm}$ as previously described (13). The MTT kit was purchased from Roche Applied Science (Indianapolis, IN).

DHE staining. The living NRVMs were stained with $10 \mu \mathrm{mol} / \mathrm{l}$ DHE (Sigma) for $30 \mathrm{~min}$ in a dark humidified chamber at $37^{\circ} \mathrm{C}$. ROS generation was indicated by red fluorescence and visualized with fluorescence microscopy. The fluorescence intensity was analyzed as previously described (13).

Statistical analysis. Data were presented as mean \pm SD from 3 independent experiments or 5 rats. Statistical analysis was carried out with Student's t test. Multiple comparisons were evaluated by ANOVA followed by Turkey's multiple-comparison test. $\mathrm{P}<0.05$ was considered as statistically significant difference.

\section{Results}

$\mathrm{H}_{2} \mathrm{O}_{2}$-induced cell viability could be reversed by $\mathrm{RHL}$ in a dose- and time-dependent manner. Firstly, primary cardiomyocytes were treated with $10 \mu \mathrm{M} \mathrm{H}_{2} \mathrm{O}_{2}$ for $24 \mathrm{~h}$. Then, the cells were incubated with $0.1,0.5,1,3,5 \mu \mathrm{M}$ RHL for $24 \mathrm{~h}$. Treatment with $10 \mu \mathrm{M} \mathrm{H}_{2} \mathrm{O}_{2}$ decreased cell viability by more than $55 \%$. In contrast, primary cardiomyocytes viability was increased by $19,25,32,42,48 \%$ with $0.1,0.5,1,3,5 \mu \mathrm{M}$ RHL incubation by in a dose- dependent manner (Fig. 1A). Meanwhile, the cells were incubated with $1 \mu \mathrm{M}$ RHL for $12,24,48,72$ h. As shown in Fig. $1 \mathrm{~B}, \mathrm{H}_{2} \mathrm{O}_{2}$ treatment decreased cardiomyocyte viability by $16,28,39,46 \%$ at $12,24,48,72 \mathrm{~h}$, repectively. However, RHL could improve $\mathrm{H}_{2} \mathrm{O}_{2}$-reduced cardiomyocyte viability by $8,15,24,28 \%$ in a time- dependent manner (Fig. 1B).

RHL reduced $R O S$ production and cell apoptosis induced by $\mathrm{H}_{2} \mathrm{O}_{2}$ treatment. DHE staining demonstrated that $\mathrm{H}_{2} \mathrm{O}_{2}$ induced the production of ROS by approximately 3.6 fold. In comparison, incubation with $1 \mu \mathrm{M}$ RHL decreased ROS production by about 2.1 fold than that of $\mathrm{H}_{2} \mathrm{O}_{2}$ (Fig. 2A). Next, we further evaluated the role of RHL in $\mathrm{H}_{2} \mathrm{O}_{2}$-induced cardiomyocytes apoptosis. TUNEL staining indicated that $\mathrm{H}_{2} \mathrm{O}_{2}$ 
A

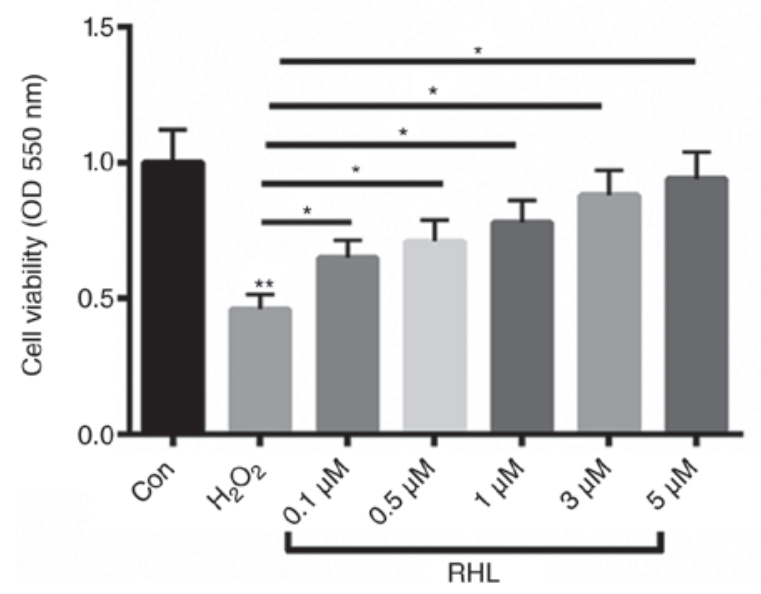

B $\quad$ Con

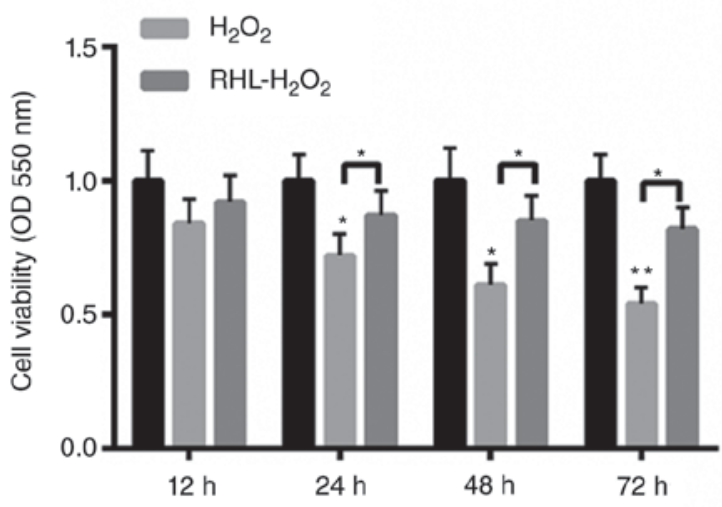

Figure $1 . \mathrm{H}_{2} \mathrm{O}_{2}$-induced cell viability could be reversed by RHL in a dose- and time-dependent manner. MTT assay of primary cardiomyocytes incubated with (A) $0.1,0.5,1,3,5 \mu \mathrm{M}$ RHL for $24 \mathrm{~h}$ or (B) $1 \mu \mathrm{M}$ RHL for $12,24,48,72 \mathrm{~h}$. ${ }^{*} \mathrm{P}<0.05,{ }^{* *} \mathrm{P}<0.01$. The symbols above comparison lines represent the comparison between the two indicated groups, and the symbols below the comparison lines compare the control with the experimental group.

A

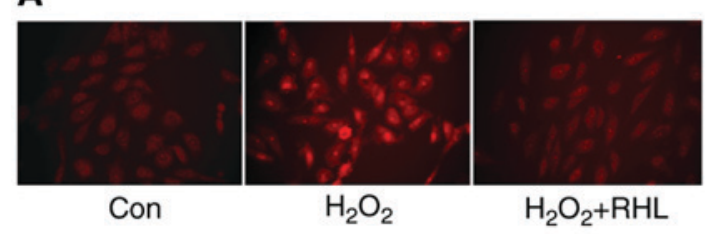

B
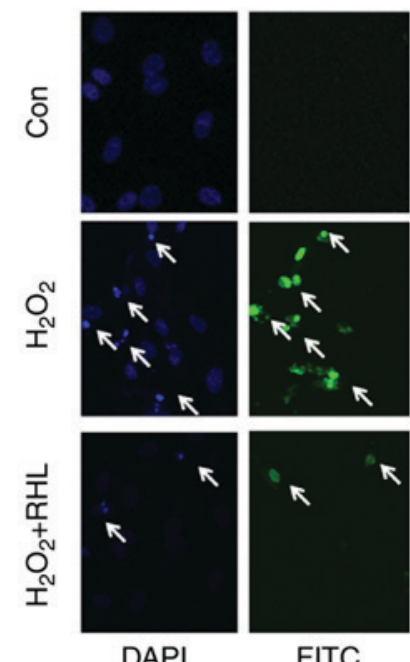

FITC
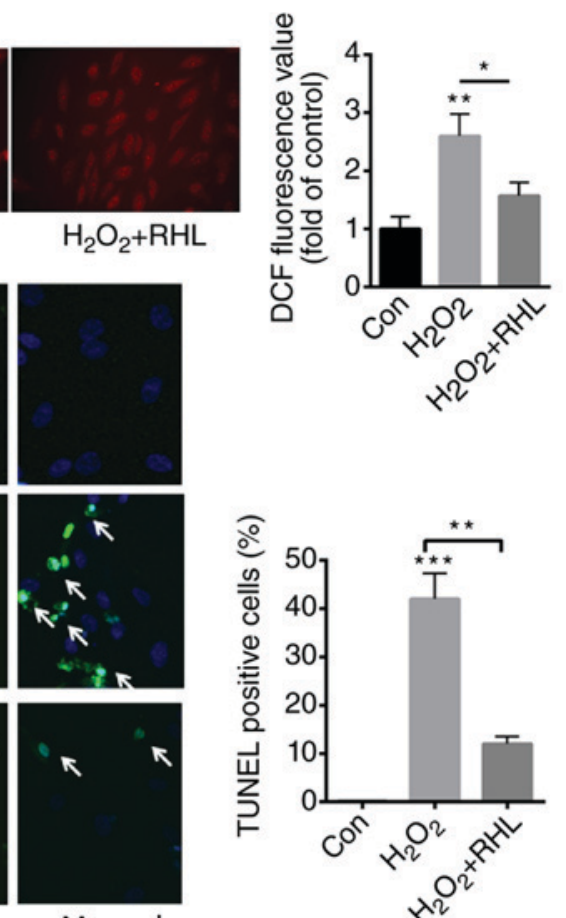

Merged

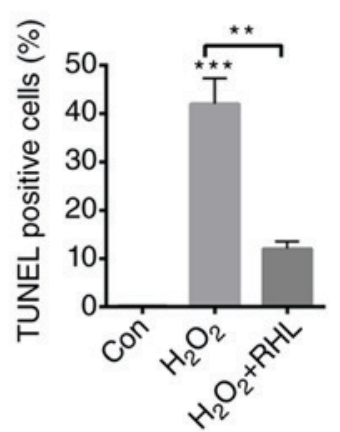

Figure 2. RHL reduced ROS production and cell apoptosis induced by $\mathrm{H}_{2} \mathrm{O}_{2}$ treatment. (A) DHE staining. (B) TUNEL staining. ${ }^{*} \mathrm{P}<0.05$, ${ }^{* *} \mathrm{P}<0.01$, ${ }^{* * * *} \mathrm{P}<0.001$. The symbols above comparison lines represent the comparison between the two indicated groups, and the symbols below the comparison lines compare the control with the experimental group.

treatment significantly increased apoptotic cells by $42 \%$ than that of normal control (0.1\%) (Fig. 2B). In comparison, RHL incubation significantly decreased $\mathrm{H}_{2} \mathrm{O}_{2}$-induced cardiomyocytes apoptosis by $30 \%$ (Fig. 2B).

RHL reduced p38MAPK signaling activation. Next, we explored the potential molecular mechanism in which RHL protects cardiomyocytes from apoptosis. We found that $\mathrm{H}_{2} \mathrm{O}_{2}$ treatment with markedly activated p38MAPK signaling by $\sim 1.12$ fold (Fig. 3). Furthermore, the pro-apoptotic protein, Bax, was significantly increased by about $\sim 1.54$ fold, while an anti-apoptotic protein, Bcl-2, was decreased by $57 \%$ (Fig. 3). In comparison, RHL markedly reduced p38MAPK signaling activation by $80 \%$. Furthermore, RHL treatment reduced the expression of Bax by $98 \%$, but increased the protein level of Bcl-2 by approximately $\sim 1$ fold (Fig. 3 ). These data indicated that RHL protected primary cardiomyocytes from $\mathrm{H}_{2} \mathrm{O}_{2}$-induced apoptosis mainly by suppressing p38MAPK activation.

The improved heart function by RHL was associated with p38MAPK signaling pathway. To further explore the effect 

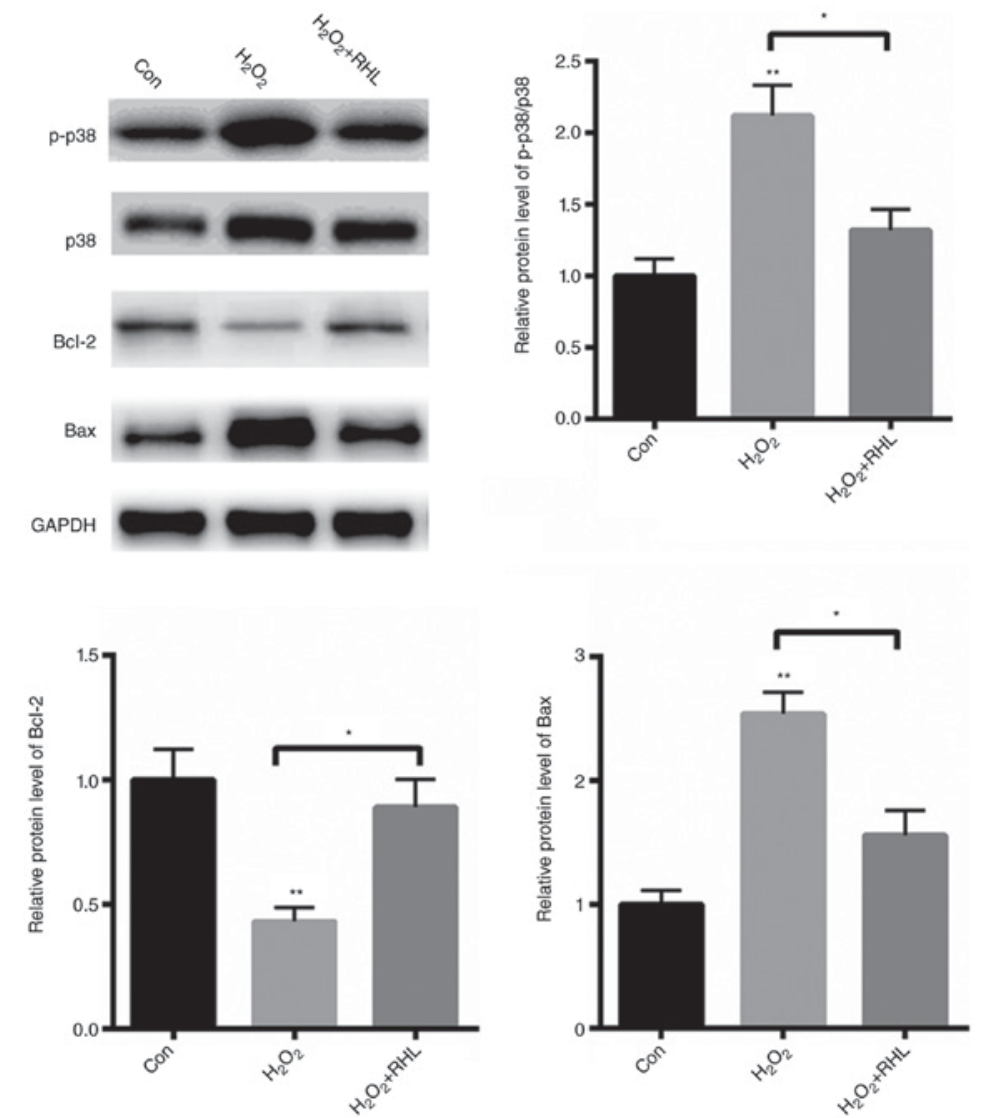

Figure 3. Western blot analysis demonstrated RHL protected primary cardiomyocytes from $\mathrm{H}_{2} \mathrm{O}_{2}$-induced apoptosis mainly by suppressing p38MAPK activation. ${ }^{~} \mathrm{P}<0.05,{ }^{* *} \mathrm{P}<0.01$. The symbols above comparison lines represent the comparison between the two indicated groups, and the symbols below the comparison lines compare the control with the experimental group.

\section{A}

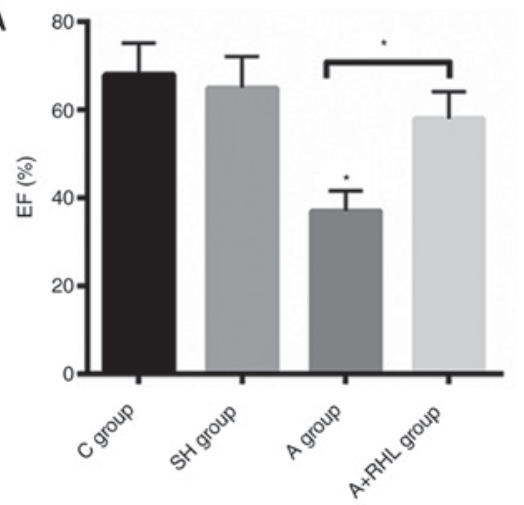

C

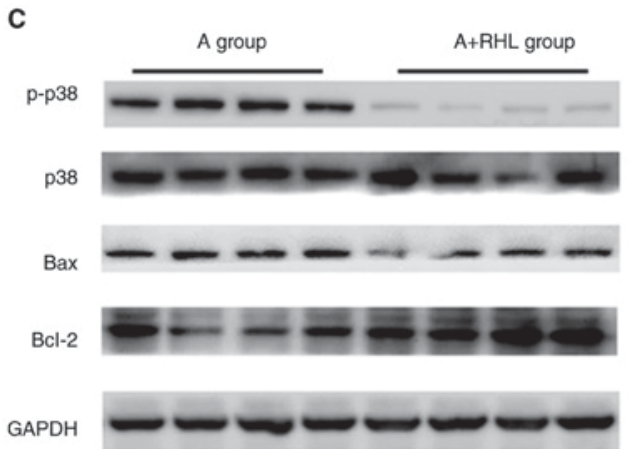

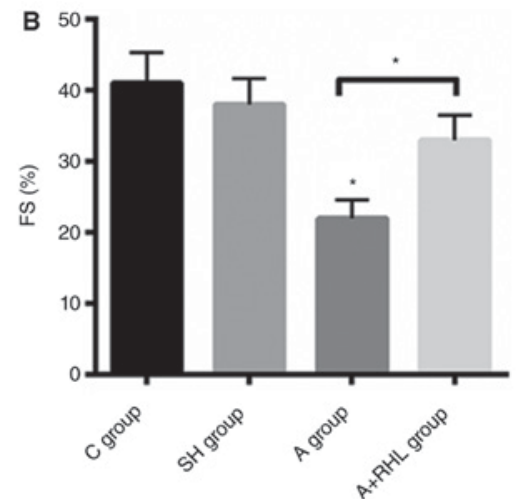

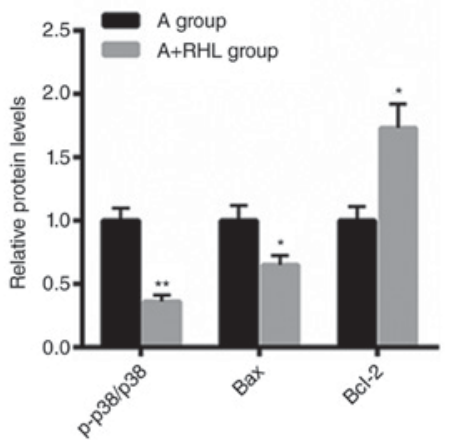

Figure 4. The improved heart function by RHL was associated with p38MAPK signaling pathway. Compared with control and sham group, coarctation of abdominal aorta group demonstrated reduced (A) ejection fraction (EF)\% and (B) fraction shortening index (FS)\%. (C) Western blot analysis of p38MAPK signaling, Bcl-2, and Bax expression after RHL treatment in HF rat models. ${ }^{*} \mathrm{P}<0.05,{ }^{* *} \mathrm{P}<0.01$. The symbols above comparison lines represent the comparison between the two indicated groups, and the symbols below the comparison lines compare the control with the experimental group. 
of RHL on heart function, echo analysis was carried out. Compared with control and sham group, coarctation of abdominal aorta group demonstrated reduced ejection fraction (EF)\% and fraction shortening index (FS)\% by 28 and $16 \%$, respectively (Fig. 4A and B). But pre-treatment with RHL markedly increased heart function by 21 and $11 \%$ than that of the operation group, respectively (Fig. 4A and B). Furthermore, we also found that RHL treatment markedly inactivated p38MAPK signaling in coarctation of abdominal aorta group. Moreover, the protein level of Bcl-2 was increased by $\sim 1.73$ fold after RHL treatment, while the expression of Bax was decreased by $35 \%$ after RHL incubation in NRVMs (Fig. 4C). These data demonstrated that RHL protects heart failure mainly by suppressing $\mathrm{p} 38 \mathrm{MAPK}$ in vivo.

\section{Discussion}

Heart failure refers to a progressive circumstance when the heart is unable to pump sufficient blood to fulfill the body's requirements at a normal filling pressure (14). The pathology includes multiple abnormities in heart muscle (15). In the past years, enhanced oxidative stress is found to be involved in the pathophysiology of congestive heart failure (CHF) (16). Clinical data have shown that patients with established CHF demonstrat increased oxidative stress markers $(8,17)$. Furthermore, the level of oxidative stress is closely related to the severity of heart failure. Thus, it is important to improve ROS production in failed hearts.

RHL has received attention for its protective role in vitro. For instance, RHL was shown to reduce inflammation and adipose infiltration in $\mathrm{KK} / \mathrm{HlJ}$ diabetic mice with non-alcoholic fatty liver disease (10). And RHL was suggested to suppress the progression of breast and ovarian cancer, hepatocellular carcinoma, cervical cancer and lung carcinoma mainly by downregulation of Bcl-2 and cyclin D expression and upregulation of BAX and Bim expression (18). In addition, RHL was demonstrated to protect the livers by reducing the expression of TNF- $\alpha$ and IL- 6 and the phosphorylation of SREBP-1c and ERK1/2 in diabetic mice (19). In the present study, we mainly evaluated the effects of RHL on failed heart. In vitro study showed that $\mathrm{H}_{2} \mathrm{O}_{2}$ treatment reduced primary cardiomyocytes viability in a time- and dose-dependent manner, while RHL could abolish the detrimental effects of $\mathrm{H}_{2} \mathrm{O}_{2}$, indicating a protective role of RHL. Further study found that $\mathrm{H}_{2} \mathrm{O}_{2}$-induced ROS production could largely be reversed by RHL. Oxidative stress is also suggested to activate cell apoptosis, thereby enhancing CHF especially in the advanced stages $(20,21)$. Then, TUNEL staining was carried out and the results showed that $\mathrm{H}_{2} \mathrm{O}_{2}$ markedly primary cardiomyocytes apoptosis. In contrast, RHL incubation decreased $\mathrm{H}_{2} \mathrm{O}_{2}$-induced cell apoptosis, indicating the protective role of RHL in oxidative stressed primary cardiomyocytes.

The mitogen-activated protein kinase p38 is an important Ser/Thr kinase that is involved in heart failure $(6,8)$. Multiple studies have been performed to explore the effects of $\mathrm{p} 38$ in heart failure $(22,23)$. In animal models, abnormal activation of p38 has been identified in heart failure. Compared with healthy heart, enhanced p38 activity is identified in the myocardial biopsies from heart failure patients $(24,25)$. In addition, in vitro studies have shown that p38 activation enhances cardiomyocyte hypertrophy, but inhibition of p38 signaling could diminish such effects (26). In line with previous studies, we found abnormal p38 activation in failed heart. More importantly, treatment with RHL could reduce p38 activation.

p38 activation is indicated as a pro-apoptotic process in cardiomyocytes (9). In Raf-1-knockout mice which is characterized by left ventricular systolic dysfunction and heart dilatation, enhanced cardiomyocyte apoptosis is identified accompanied with an increase in p38 kinase activity and cell apoptosis (27). Furthermore, upregulation of p38 $\alpha$ in cultured neonatal cardiomyocytes (28) and expression of transforming growth factor- $\beta$-activated kinase- 1 are related to significant cardiac apoptosis in the mouse heart (29). In the current study, we found that treatment of RHL significantly enhanced the expression of anti-apoptotic protein, Bcl-2, but markedly reduced the protein level of Bax in primary cardiomyocytes, indicating its anti-apoptotic role in the cardiac setting.

In summary, RHL protects heart failure mainly by reducing ROS production and cardiomyocyte apoptosis through reducing p38MAPK activation.

\section{References}

1. Suzuki H, Nodera M, Kamioka M, Kaneshiro T, Kamiyama Y and Takeishi Y: Intracardiac impedance after cardiac resynchronization therapy is a novel predictor for worsening of heart failure. Heart Vessels 32: 926-931, 2017.

2. Senni M, D'Elia E, Emdin M and Vergaro G: Biomarkers of heart failure with preserved and reduced ejection fraction. Handb Exp Pharmacol 243: 79-108, 2017.

3. Pecoraro A, Crescenzi L, Carucci L, Genovese A and Spadaro G: Heart failure not responsive to standard immunosuppressive therapy is successfully treated with high dose intravenous immunoglobulin therapy in a patient with eosinophilic granulomatosis with polyangiitis (EGPA). Int Immunopharmacol 45: 13-15, 2017.

4. Marx N: Heart failure and diabetes-underestimated, underdiagnosed and poorly understood: A call for action. Diab Vasc Dis Res 14: 67-68, 2017.

5. Mathur A, Fernández-Avilés F, Dimmeler S, Hauskeller C, Janssens S, Menasche P, Wojakowski W, Martin JF and Zeiher A; BAMI Investigators: The consensus of the Task Force of the European Society of Cardiology concerning the clinical investigation of the use of autologous adult stem cells for the treatment of acute myocardial infarction and heart failure: Update 2016. Eur Heart J, Feb 15, 2017 (Epub ahead of print).

6. Arabacilar P and Marber M: The case for inhibiting p38 mitogen-activated protein kinase in heart failure. Front Pharmacol 6: 102, 2015.

7. Cook SA, Sugden PH and Clerk A: Activation of c-Jun N-terminal kinases and p38-mitogen-activated protein kinases in human heart failure secondary to ischaemic heart disease. J Mol Cell Cardiol 31: 1429-1434, 1999.

8. Di Lisa F, Kaludercic N and Paolocci N: $\beta_{2}$-Adrenoceptors, NADPH oxidase, ROS and p38 MAPK: Another 'radical' road to heart failure? Br J Pharmacol 162: 1009-1011, 2011.

9. Marber MS, Rose B and Wang Y: The p38 mitogen-activated protein kinase pathway-a potential target for intervention in infarction, hypertrophy, and heart failure. J Mol Cell Cardiol 51: 485-490, 2011.

10. Wei J, Zhen YZ, Cui J, He FL, Shen T, Hu G, Ren XH and Lin YJ: Rhein lysinate decreases inflammation and adipose infiltration in KK/HIJ diabetic mice with non-alcoholic fatty liver disease. Arch Pharm Res 39: 960-969, 2016.

11. Lin YJ, Zhen YZ, Wei J, Liu B, Yu ZY and Hu G: Effects of Rhein lysinate on $\mathrm{H} 2 \mathrm{O} 2$-induced cellular senescence of human umbilical vascular endothelial cells. Acta Pharmacol Sin 32: 1246-1252, 2011

12. Lin YJ, Zhen YZ, Zhao YF, Wei J and Hu G: Rhein lysinate induced $S$-phase arrest and increased the anti-tumor activity of 5-FU in HeLa cells. Am J Chin Med 39: 817-825, 2011. 
13. Hannan RD, Luyken J and Rothblum LI: Regulation of ribosomal DNA transcription during contraction-induced hypertrophy of neonatal cardiomyocytes. J Biol Chem 271: 3213-3220, 1996.

14. Song B, Li T, Chen S, Yang D, Luo L, Wang T, Han X, Bai L and Ma A: Correlations between MTP and ros levels of peripheral blood lymphocytes and readmission in patients with chronic heart failure. Heart Lung Circ 25: 296-302, 2016

15. Rojas A, Mercadal E, Figueroa $\mathrm{H}$ and Morales MA: Advanced Glycation and ROS: A link between diabetes and heart failure. Curr Vasc Pharmacol 6: 44-51, 2008.

16. Astashkin EI, Glezer MG, Vinokurov MG, Egorova ND, Orekhova NS, Novikova AN, Grachev SV, Yurinskaya MM and Sobolev KE: Actovegin reduces the ROS level in blood samples of heart failure patients and diminishes necrosis of SK-N-SH human neuroblastoma cells. Dokl Biol Sci 448: 57-60, 2013.

17. Borchi E, Bargelli V, Stillitano F, Giordano C, Sebastiani M, Nassi PA, d'Amati G, Cerbai E and Nediani C: Enhanced ROS production by NADPH oxidase is correlated to changes in antioxidant enzyme activity in human heart failure. Biochim Biophys Acta 1802: 331-338, 2010.

18. Liu J, Zhang K, Zhen YZ, Wei J, Hu G, Gao JL, Tian YX and Lin YJ: Antitumor activity of rhein lysinate against human glioma U87 cells in vitro and in vivo. Oncol Rep 35: 1711-1717, 2016.

19. Lin YJ, Hu G, Li KJ, Zhao YF, Wei J and Zhen YZ: The protection of Rhein lysinate to liver in diabetic mice induced by high-fat diet and streptozotocin. Arch Pharm Res 38: 885-892, 2015.

20. Hafstad AD, Nabeebaccus AA and Shah AM: Novel aspects of ROS signalling in heart failure. Basic Res Cardiol 108: 359, 2013.

21. Zuo L, Chuang CC, Hemmelgarn BT and Best TM: Heart failure with preserved ejection fraction: Defining the function of ROS and NO. J Appl Physiol (1985) 119: 944-951, 2015.

22. Heusch P, Canton M, Aker S, van de Sand A, Konietzka I, Rassaf T, Menazza S, Brodde OE, Di Lisa F, Heusch G and Schulz R: The contribution of reactive oxygen species and p38 mitogen-activated protein kinase to myofilament oxidation and progression of heart failure in rabbits. Br J Pharmacol 160 $1408-1416,2010$.
23. Hoefer J, Azam MA, Kroetsch JT, Leong-Poi H, Momen MA, Voigtlaender-Bolz J, Scherer EQ, Meissner A, Bolz SS and Husain M: Sphingosine-1-phosphate-dependent activation of p38 MAPK maintains elevated peripheral resistance in heart failure through increased myogenic vasoconstriction. Circ Res 107: 923-933, 2010.

24. Kerkela R and Force T: p38 mitogen-activated protein kinase: A future target for heart failure therapy? J Am Coll Cardiol 48: $556-558,2006$.

25. Kita T, Ogawa M, Sato H, Kasai K, Tanaka T and Tanaka N: Role of p38 mitogen-activated protein kinase pathway on heart failure in the infant rat after burn injury. Int J Exp Pathol 89: 55-63, 2008.

26. Nemoto S, Sheng Z and Lin A: Opposing effects of Jun kinase and p38 mitogen-activated protein kinases on cardiomyocyte hypertrophy. Mol Cell Biol 18: 3518-3526, 1998.

27. Irukayama-Tomobe Y, Miyauchi T, Kasuya Y, Sakai S, Goto K and Yamaguchi I: Activation of peroxisome proliferator-activated receptor-alpha decreases endothelin-1-induced p38 mitogen-activated protein kinase activation in cardiomyocytes. J Cardiovasc Pharmacol 44 (Suppl 1): S358-S361, 2004.

28. Inagaki K, Satoh T, Yagi-Utsumi M, Le Gulluche AC, Anzai T, Uekusa Y, Kamiya Y and Kato K: Redox-coupled structural changes of the catalytic a' domain of protein disulfide isomerase. FEBS Lett 589: 2690-2694, 2015.

29. Zhang D, Gaussin V, Taffet GE, Belaguli NS, Yamada M, Schwartz RJ, Michael LH, Overbeek PA and Schneider MD: TAK1 is activated in the myocardium after pressure overload and is sufficient to provoke heart failure in transgenic mice. Nat Med 6: $556-563,2000$. 